\title{
Observation of a four-electron Auger process in near- $K$-edge photoionization of singly charged carbon ions
}

\author{
A. Müller, ${ }^{1, *}$ A. Borovik Jr.,${ }^{1}$ T. Buhr,${ }^{1,2}$ J. Hellhund, ${ }^{1}$ K. Holste,${ }^{1}$ A. L. D. Kilcoyne, ${ }^{3}$ \\ S. Klumpp,${ }^{4}$ M. Martins,${ }^{4}$ S. Ricz,${ }^{1,5}$ J. Viefhaus,${ }^{6}$ and S. Schippers ${ }^{1}$ \\ ${ }^{1}$ Institut für Atom- und Molekülphysik, Justus-Liebig-Universität Giessen, Germany \\ ${ }^{2}$ Physikalisch-Technische Bundesanstalt, Braunschweig, Germany \\ ${ }^{3}$ Advanced Light Source, Lawrence Berkeley National Laboratory, Berkeley, California, USA \\ ${ }^{4}$ Institut für Experimentalphysik, Universität Hamburg, Germany \\ ${ }^{5}$ Institute of Nuclear Research of the Hungarian Academy of Sciences, Debrecen, Hungary \\ ${ }^{6}$ FS-PE, DESY, Hamburg, Germany
}

(Dated: November 21, 2014)

\begin{abstract}
Single, double and triple ionization of $\mathrm{C}^{1+}$ ions by single photons have been investigated in the energy range 286 to $326 \mathrm{eV}$, i.e., in the range from the lowest-energy $K$-vacancy resonances to well beyond the $K$-shell ionization threshold. Clear signatures of $\mathrm{C}^{1+}\left(1 s 2 s^{2} 2 p^{2}{ }^{2} D,{ }^{2} P\right)$ resonances have been found in the triple-ionization channel. The only possible mechanism producing $\mathrm{C}^{4+}\left(1 s^{2}\right)$ via these resonances is direct triple-Auger decay, i.e., a four-electron process with simultaneous emission of three electrons.
\end{abstract}

PACS numbers: 32.80.Aa,32.80.Fb,32.80.Hd,32.80.Zb

Multi-electron processes mediated by electron-electron interactions have attracted general interest ever since the early days of quantum mechanics [1]. The free helium atom provides the minimum number of electrons necessary for electron correlation effects to occur which crucially determine the atomic structure of any He-like system and the decay probabilities of its excited states [2]. The most important decay mechanism of doubly excited He or, more general, atomic systems with two or more electrons is the Auger process in which two electrons exchange energy so that one drops into a lower bound state while the second electron escapes to the continuum carrying away the transition-characteristic excess energy (see Fig. 1a). Likewise, double ionization of a He-like system by an incident photon is seriously affected by electron correlation [3].

The next level of higher-order processes is reached when three electrons interact with one another either when bound in an atom or when two bound electrons interact with one impinging from outside [4]. Carlson and Krause [5] identified double-Auger processes (see Fig. 1b). Alternatively, in a hollow ion with an empty $K$ shell a three-electron process is possible with two electrons dropping down into the $K$ shell and one electron being ejected into the continuum [6]. Three-electron correlations also govern the processes of trielectronic capture $[7,8]$ and triple ionization by a single photon $[9,10]$.

Even the four-electron process of quadruelectronic recombination has been observed in which three bound electrons are excited while an incident electron is captured [11]. Accordingly, there should also be a fourelectron interaction mechanism in the sequence of singleand double-Auger processes, the direct triple-Auger decay (see Fig. 1c). Clear identification of such triple-Auger decay by electron-spectroscopy as performed for double-
Auger decay [12] would require population of a suitable inner-shell-vacancy state and then the simultaneous detection of all three ejected electrons and measurement of their energies, the sum of which has to match the transition energy of the electron that remains bound. Experiments based on multi-electron coincidence spectroscopy have reached a very high degree of sophistication and provide a wealth of information about energy correlations between ejected electrons from multi-electron atoms $([13,14]$ and references therein). Nevertheless, direct triple-Auger decay with simultaneous emission of three electrons has never been unambiguously observed.

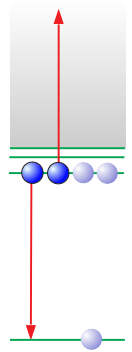

(a)

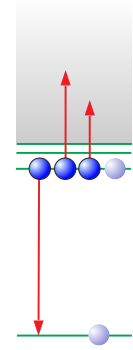

(b)

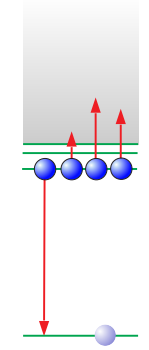

(c)
FIG. 1. (color online) Single- (a), double- (b) and tripleAuger (c) decay of a K-shell excited B-like atom.

The minimum number of electrons required to observe triple-Auger decay in an atom is four (Fig. 1c). A configuration with a $K$ vacancy and four electrons in the $L$ shell can be prepared by term-selective $K \rightarrow L$ photoexcitation of a B-like atom or ion such as $\mathrm{C}^{1+}$. The final charge state after triple-Auger decay is +4 with both remaining electrons in the $K$ shell. Any cascade process would leave at least one electron in the $L$ shell. Detecting the pro- 


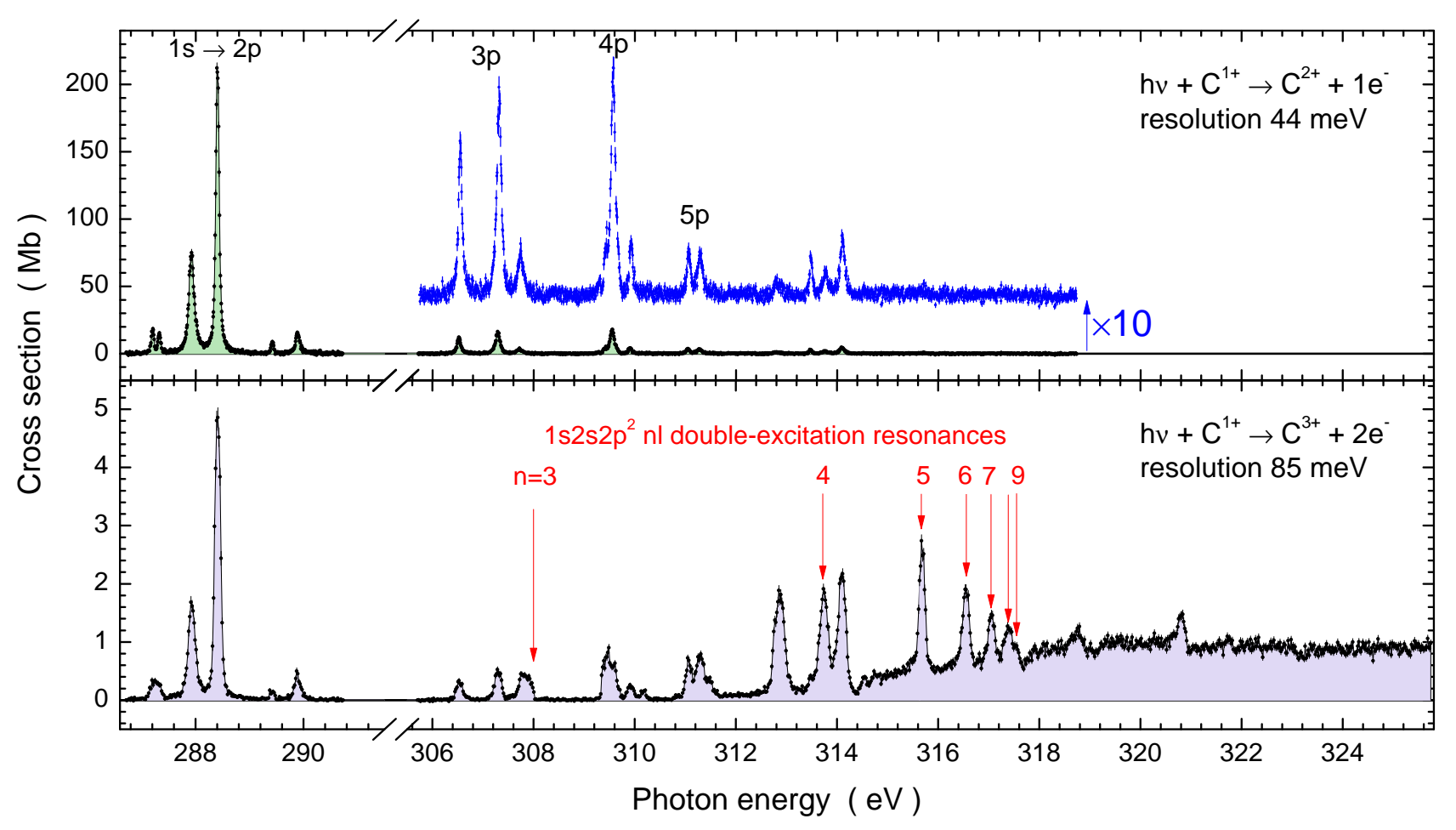

FIG. 2. (color online) Absolute cross sections for single and double ionization of $\mathrm{C}^{1+}$ ions by single photons. The photon energy axis has a break in the range where no resonances are expected. The experimental data are shown as small dots with statistical error bars. They are connected by a solid line with shading. The cross section data for single ionization in the high-energy region were multiplied by a factor of 10 and displayed again with a vertical offset. Important resonance groups are identified by their configurations.

duction of heliumlike $\mathrm{C}^{4+}$ ions after $K$-shell excitation of $\mathrm{C}^{1+}$ thus has the potential of unambiguously identifying the direct triple-Auger process.

Photoexcitation and resulting ionization of B-like ions has been investigated by experiments previously [15-19]. All photoionization experiments with ionic targets involving $K$-vacancy production have been limited so far to the strongest resonance features in the spectrum and, more important in the present context, all these experiments were restricted to the single-ionization channel.

Here we report absolute cross section measurements for single, double and triple ionization of $\mathrm{B}$-like $\mathrm{C}^{1+}$ ions in the photon energy range $286-326 \mathrm{eV}$. The experiments were carried out at the newly developed photonion merged-beam setup PIPE (photon-ion spectrometer at PETRA III) [20] using monochromatized undulator radiation from the PETRA III synchrotron light source in Hamburg. With the extended capabilities of our new experiment, structures and processes in ionized atoms, molecules and clusters can be observed which have not been previously accessible to experiments.

The experimental arrangement and procedures have been described in detail recently [20]. In short, ions produced from $\mathrm{CH}_{4}$ gas in an electron-cyclotron-resonance (ECR) ion source were accelerated by $6 \mathrm{kV}$, magnetically analyzed to obtain a pure ${ }^{12} \mathrm{C}^{1+}$ ion beam which was then transported to the interaction region, collimated and merged with the circularly polarized photon beam available at beamline P04 of PETRA III. The product ions were separated from the parent ion beam by a dipole magnet (with flux density B2) inside which the primary beam was collected in a large Faraday cup. The photoionized ions were passed through a spherical 180-degree out-of-plane deflector (which requires different settings for different charge states) to suppress background from stray electrons, photons and ions and then entered a single-particle detector with near-100\% detection efficiency. In the present experiment the $\mathrm{C}^{2+}, \mathrm{C}^{3+}$, and $\mathrm{C}^{4+}$ products were found at $\mathrm{B} 2=157.6 \mathrm{mT}, 210.5 \mathrm{mT}$, and $316.0 \mathrm{mT}$ with peak widths between $0.8 \mathrm{mT}$ and $1.5 \mathrm{mT}$, respectively. At this level of separation "cross talk" between product channels can be totally excluded. The high brightness and flux of the photon beam $\left(2 \times 10^{11} \mathrm{~s}^{-1}\right.$ at $288 \mathrm{eV}$ energy and $16 \mathrm{meV}$ bandwidth) permitted tight collimation of the ion beam (1.5 nA through an area of $1 \mathrm{~mm} \times 0.2 \mathrm{~mm}$ at the entrance to the interaction region with a divergence of less than $3 \mathrm{mrad}$ ) and, hence, a significantly improved spatial overlap with the photon beam compared to earlier experiments.

The photon flux was measured with a calibrated photodiode. The photon energy scale was calibrated with an uncertainty of better than $\pm 30 \mathrm{meV}$ by remeasuring 
known resonances in $\mathrm{C}^{3+}[21]$ at $9 \mathrm{meV}$ resolution (corresponding to a resolving power of 38000 ). Doppler shifts due to the ion velocity directed towards the incoming photons were corrected for. The systematic absolute uncertainty of the measured cross sections for single and double ionization is $\pm 15 \%$ [20]. In the case of triple ionization the cross section was inferred from ratios between consecutive recordings of the spectra for double and triple ionization with the experimental conditions unchanged, and by normalizing the triple-ionization yield to the absolute data for double ionization using the measured ratios. An estimate of the resulting uncertainty of the extremely small absolute triple-ionization cross section is $\pm 50 \%$. Since the focus of this paper is on higher-order processes and particularly on the direct triple-Auger decay, detailed results for $1 s \rightarrow 2 p$ transitions in single ionization obtained with $16 \mathrm{meV}$ resolution will be presented in a separate publication.

Figure 2 shows complete spectra of single and double ionization by single photons. The single-ionization spectrum with absolute cross sections was taken at a resolution of $44 \mathrm{meV}$ yielding high statistical precision for the strongest resonance group associated with $1 s \rightarrow 2 p$ transitions. The cross sections of the resonances associated with excitations to higher $n p$ subshells are smaller by more than an order of magnitude compared to those of the $1 s \rightarrow 2 p$ transitions. Although the measurements extend well beyond the $K$-shell ionization threshold there is no signature of the $K$-edge in the single-ionization cross section. This is not a surprise since the removal of a $K$ shell electron produces an autoionizing state which decays predominantly by Auger processes. Therefore, contributions from $K$-shell ionization have to be expected in the cross sections for multiple ionization.

The lower panel of Fig. 2 shows the cross section for double ionization measured at $85 \mathrm{meV}$ resolution. The ordinate scale is almost two orders of magnitude smaller than that of the upper panel. The resonances present in single ionization also appear in double ionization although with different relative sizes depending on the photon energy. Different from single ionization the highenergy part of the cross section becomes relatively much more important. Obviously the resonances with one electron in a state with high principal quantum numbers $n$ and even more so the $K$-shell-ionized ions end up as $\mathrm{C}^{3+}$ products after double- or single-Auger decay, respectively. We note that the Rydberg sequence of resonances dominating double ionization at energies above $315 \mathrm{eV}$ is assigned to double-excitations of ground-state $\mathrm{C}^{1+}$ ions to $\left(1 s 2 s 2 p^{2} n l\right)$ configurations with the principal quantum numbers $n$ indicated in Fig. 2.

The results discussed so far illustrate the technological progress realized with the PIPE setup [20] at the most brilliant synchrotron source worldwide. Significant improvement of experimental conditions could be achieved in comparison with all previous experiments addressing

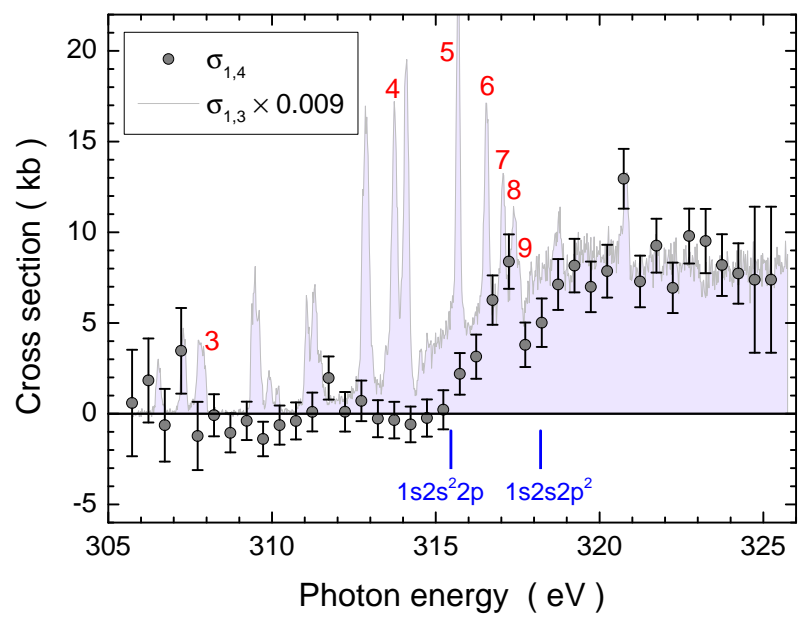

FIG. 3. (color online) Cross sections for triple ionization (shaded circles) of $\mathrm{C}^{1+}$ ions by single photons at energies around the $K$ edge. The energy resolution was $130 \mathrm{meV}$ in this case. Also shown is the cross section (from Fig. 2) for double ionization multiplied by a factor 0.009 (gray solid line with light shading). Members of the $1 s 2 s 2 p^{2} n l$ Rydberg sequence are identified by their principal quantum number $n$. The lowest threshold for $K$-shell ionization (producing $1 s 2 s^{2} 2 p^{3} P$ levels was calculated using the Cowan Code [22]. It is shown as a (blue) vertical bar at $315.46 \mathrm{eV}$. The $1 s 2 s 2 p^{2} n l$ Rydberg series converges to the series limit indicated by the (blue) vertical bar at $318.2 \mathrm{eV}$.

photoionization of ions near the $\mathrm{K}$ edge. As a result of these advancements completely new territory of research becomes accessible.

By opening the monochromator slits a maximum photon flux of $1.2 \times 10^{13} \mathrm{~s}^{-1}$ at $315 \mathrm{eV}$ with $130 \mathrm{meV}$ bandwidth became available. At that flux, triple ionization of $\mathrm{C}^{1+}$ was observed (see Fig. 3) in the region of the $K$ edge. Also shown in Fig. 3 are the double-ionization cross sections from Fig. 2 multiplied by a factor 0.009 that brings the data measured above the $K$ edge to the scale of triple ionization. Although the statistical precision of the triple-ionization data is quite limited one can recognize that the resonances below the $1 s 2 s^{2} 2 p$ threshold do not significantly contribute to the triple-ionization signal. Even the production of $\mathrm{C}^{4+}$ after removal of a $K$-shell electron leaving an intermediate $1 s 2 s^{2} 2 p^{3} P K$ vacancy state appears to be suppressed. Signal below the next threshold for the $1 s 2 s 2 p^{2}$ configuration may just be due to sequential electron emission processes involving a $2 p \rightarrow 2 s$ Coster-Kronig decay of the $1 s 2 s 2 p^{2} n l$ resonant states with $n \geq 5$ in the first step and a $K-L L$ Auger transition in the second.

For the strongest resonances found in single ionization, additional measurements of single, double and triple ionization were carried out at energy resolution $92 \mathrm{meV}$. The lowest panel in Fig. 4 shows the unambiguous observation of $\mathrm{C}^{4+}$ product ions arising from $K$-shellexcited $\mathrm{C}^{1+}\left(1 s 2 s^{2} 2 p^{2}{ }^{2} D,{ }^{2} P\right)$ states. The photon ener- 


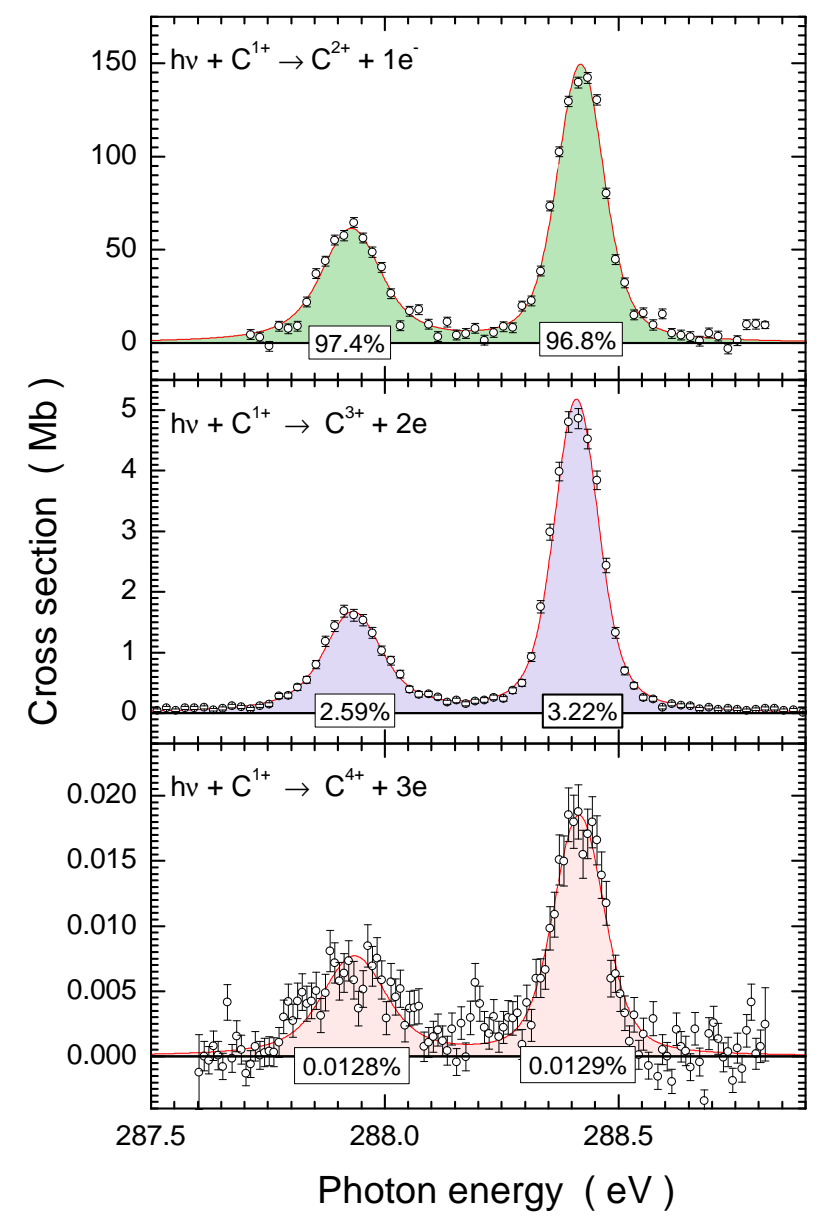

FIG. 4. (color online) Cross section for single, double and triple ionization of ground-state $\mathrm{C}^{1+}$ ions by single photons with $92 \mathrm{meV}$ bandwidth. The resonances seen in all the observed channels are associated with $K$-shell-excited $\mathrm{C}^{1+}\left(1 s 2 s^{2} 2 p^{2}{ }^{2} D,{ }^{2} P\right)$ terms. The percentages given for the areas of the peaks indicate the branching factors for single-, double-, and triple-Auger decay.

gies associated with these two resonances are characteristic for transitions from the ground-state term of $\mathrm{C}^{1+}$ : $\gamma+\mathrm{C}^{1+}\left(1 s^{2} 2 s^{2} 2 p^{2} P\right) \rightarrow \mathrm{C}^{1+}\left(1 s 2 s^{2} 2 p^{2}{ }^{2} D,{ }^{2} P\right)$. The resulting $K$-vacancy states can then obviously decay by the emission of up to three electrons. We argue that the only possible process in the particular case of a five-electron system with a $K$ vacancy and four electrons in the energetically lowest $L$-shell term $\mathrm{C}^{1+}\left(1 s 2 s^{2} 2 p^{2}{ }^{2} D,{ }^{2} P\right)$ is the direct triple-Auger decay which involves correlated interaction of four electrons. Clearly, one must consider other possible mechanisms that can potentially produce the observed $\mathrm{C}^{4+}$ product ions. Questions to be discussed are: What is the probability for a parent $\mathrm{C}^{1+}$ ion to absorb two photons both resonant with the transition $\mathrm{C}^{1+}\left(1 s^{2} 2 s^{2} 2 p{ }^{2} P\right) \rightarrow \mathrm{C}^{1+}\left(1 s 2 s^{2} 2 p^{2}{ }^{2} D,{ }^{2} P\right)$ and what is the probability of a $\mathrm{C}^{1+}$ parent ion to loose electrons by absorption of one resonant photon and to loose at least one more electron in a subsequent residual-gas collision?
The photon beam in a synchrotron has a pulse structure. In the present case the duty cycle $\eta$ was about $3.5 \times 10^{-4}$. On the dominant resonance at $288.4 \mathrm{eV}$ with a cross section of about $150 \mathrm{Mb}$ the instantaneous singleionization probability per ion was $P_{1}=R_{1} /\left(I_{i} \eta\right)=$ $1.6 \times 10^{-4}$ during a photon pulse with $R_{1}$ the measured $\mathrm{C}^{2+}$ product ion count rate and, in the present case, $I_{i}=4 \times 10^{11} \mathrm{~s}^{-1}$ the incident $\mathrm{C}^{1+}$ ion flux. Absorption of a second photon is non-resonant because the energy of the second photon is the same as that of the first, while the ion has changed its electronic structure and probably already also its charge state. Off resonance, the cross sections are less than $0.2 \mathrm{Mb}$ which reduces the probability for the absorption of two photons to the level of $P_{2}=0.2 / 150 \times 0.5 P_{1}^{2} \leq 1.7 \times 10^{-11}$ during photon pulses. The resulting time-averaged count rate from such events is near $P_{2} \eta I_{i}=0.0024 \mathrm{~s}^{-1}$, i.e., negligible in comparison with the count rate $\left(\approx 5 \mathrm{~s}^{-1}\right)$ observed for triple ionization.

The second scenario to be considered is photoabsorption and subsequent electron loss in a residual-gas collision. Starting with an electron-loss collision would produce a non-resonant situation for the fixed-energy photons. Two-electron loss in an ion-atom collision at $6 \mathrm{keV}$ energy can be neglected. Thus, only a reaction chain consisting of photoabsorption plus two-electron emission plus electron-loss collision in the residual gas could possibly result in net triple ionization. The electron loss should then occur from an intermediate $\mathrm{C}^{3+}$ ion. The probability for a $\mathrm{C}^{3+}$ ion to loose an electron is certainly smaller than that for a $\mathrm{C}^{1+}$ ion. For an estimate of this latter probability the background count rate observed in the $\mathrm{C}^{1+}$ single-ionization channel may be totally attributed to electron loss in residual-gas collisions. That background per incident ion, i.e., the one-electron loss probability, was $8 \times 10^{-8}$ which can be considered a conservative upper limit for the related step in the envisaged reaction chain. The measured probability for producing $\mathrm{C}^{3+}$ on the photoabsorption resonance (see Fig. 4) is less than $8 \times 10^{-10}$ leaving a combined probability of $6.4 \times 10^{-17}$ for producing $\mathrm{C}^{4+}$ from $\mathrm{C}^{1+}$ in a reaction chain involving photoabsorption, two-electron emission, and an ionizing residual-gas collision. The resulting count rate is estimated to be at most $0.0002 \mathrm{~s}^{-1}$.

Therefore we conclude that our observation of $\mathrm{C}^{4+}$ product ions can only result from the interaction of a single photon with a ground-state $\mathrm{C}^{1+}$ ion. The energy (about $288 \mathrm{eV}$ ) stored in the $K$-shell-excited intermediate $\mathrm{C}^{1+}\left(1 s 2 s^{2} 2 p^{2}{ }^{2} D,{ }^{2} P\right)$ ion can only produce ionization if the $K$-shell vacancy is filled by one of the L-shell electrons during the decay process. This requires that all three additional electrons originally residing in the $L$ shell must have been ejected in a single, correlated four-electron process, the direct triple-Auger decay, which leaves only two electrons in the detected $\mathrm{C}^{4+}$ product ions and both these electrons must be in the $K$ shell. Thus the present ex- 
periment unambiguously demonstrates the existence of direct triple-Auger decay for the first time.

The matrix elements associated with double-Auger decay have been formulated by Amusia et al. [23] showing the complexity of this three-body process. For tripleAuger decay no theoretical predictions are available yet. However, on the basis of our experiment we have been able to quantify the probability of four-body Auger decay of well prepared excited levels in $\mathrm{C}^{1+}$ ions. The observed ratios of triple- to double- to single-Auger decay rates are of the order of $10^{-4}: 10^{-2}: 1$.

The clear signals and quantitative information on branching ratios obtained for the triple-Auger decay with simultaneous emission of 3 electrons provide an ideal testbed for studies of the four-body-Coulomb problem ([10] and references therein) where the correlated dynamics of three unbound electrons in the external field of the remaining closed-shell ion is not obscured by indirect (sequential) processes. Besides its fundamental character the observed mechanism has many practical implications. It constitutes a new way of producing, for example, He-like carbon ions in dilute astrophysical environments, such as the interstellar medium, weakly irradiated and ionized in the K-shell by short-wavelength photons. While the branching ratio for triple-Auger decay is only of the order of $10^{-4}$ for a $K$-shell excited $\mathrm{C}^{1+}$ ion, it is most likely much larger for systems with many electrons residing in identical subshells. Thus, our findings are important for all fields where ejection of electrons is a central mechanism and where multi-electron processes determine the charge-state distribution of atomicscale particles. A timely example is multiphoton excitation by free-electron lasers (FEL) ([24] and references therein). The triple-Auger mechanism may help to better understand the complex deexcitation processes following multi-excitation of atoms by intense FEL pulses. We also mention the very active research field of interatomic and intermolecular Coulombic decay ([25] and references therein) which shows interesting parallels to the present results.

This work was made possible by grants from Bun- desministerium für Bildung und Forschung and from Deutsche Forschungsgemeinschaft. The Giessen team acknowledges additional support from the Hesse State Initiative for the Development of Scientific and Economic Excellence (LOEWE) in the LOEWE-Projects ELCH and RITSAT. We thank the P04 beamline team for their support of our work.

* Alfred.Mueller@iamp.physik.uni-giessen.de

[1] W. Heisenberg, Z. Physik 39, 499 (1926).

[2] G. Tanner, K. Richter, and J.-M. Rost, Rev. Mod. Phys. 72, 497 (2000).

[3] A. Knapp et al., Phys. Rev. Lett. 89, 033004 (2002).

[4] A. Müller, Adv. At. Mol. Phys. 55, 293 (2008).

[5] T. A. Carlson and M. O. Krause, Phys. Rev. Lett. 14, 390 (1965).

[6] E. De Filippo, G. Lanzanò, H. Rothard, and C. Volant, Phys. Rev. Lett. 100, 233202 (2008).

[7] A. Müller et al., Phys. Rev. Lett. 63, 758 (1989).

[8] M. Schnell et al., Phys. Rev. Lett. 91, 043001 (2003).

[9] R. Wehlitz et al., Phys. Rev. Lett. 81, 1813 (1998).

[10] J. Colgan, A. Emmanouilidou, and M. S. Pindzola, Phys. Rev. Lett. 110, 063001 (2013).

[11] C. Beilmann et al., Phys. Rev. Lett. 107, 143201 (2011).

[12] J. Viefhaus et al., Phys. Rev. Lett. 92, 083001 (2004).

[13] P. Lablanquie et al., J. Electron Spectrosc. 156, 51 (2007).

[14] Y. Hikosaka et al., Phys. Rev. Lett. 107, 113005 (2011).

[15] H. Kjeldsen et al., Astrophys. J. 524, L143 (1999).

[16] H. Kjeldsen et al., Astrophys. J. Suppl. Ser. 135, 285 (2001).

[17] A. S. Schlachter et al., J. Phys. B 37, L103 (2004).

[18] M. F. Gharaibeh et al., J. Phys. B 47, 065201 (2014).

[19] B. M. McLaughlin et al., J. Phys. B 47, 115201 (2014).

[20] S. Schippers et al., J. Phys. B 47, 115602 (2014).

[21] A. Müller et al., J. Phys. B 42, 235602 (2009).

[22] R. D. Cowan, The Theory of Atomic Structure and Spectra (University of California Press, Berkeley, 1981).

[23] M. Y. Amusia, I. S. Lee, and V. A. Kilin, Phys. Rev. A 45, 4576 (1992).

[24] H. Fukuzawa et al., Phys. Rev. Lett. 110, 173005 (2013).

[25] K. Gokhberg et al., Nature 505, 661 (2014). 